\title{
A Novel Energy Aware Clustering Technique for Routing in Wireless Sensor Networks
}

\author{
Ouadoudi Zytoune $^{1}$, Youssef Fakhri ${ }^{2}$, Driss Aboutajdine ${ }^{1}$ \\ ${ }^{1}$ LRIT unite associée au CNRST, Faculty of Sciences Agdal, Rabat, Morocco \\ ${ }^{2}$ University Ibn Tofail, Kenitra, Morocco \\ E-mail:zytoune@gmail.com,yousseffakhri@yahoo.fr,aboutaj@fsr.ac.ma \\ Received December 20, 2009; revised December 28, 2009; accepted December 30, 2009
}

\begin{abstract}
Cluster-based architectures are one of the most practical solutions in order to cope with the requirements of large-scale wireless sensor networks (WSN). Cluster-head election problem is one of the basic QoS requirements of WSNs, yet this problem has not been sufficiently explored in the context of cluster-based sensor networks. Specifically, it is not known how to select the best candidates for the cluster head roles. In this paper, we investigate the cluster head election problem, specifically concentrating on applications where the energy of full network is the main requirement, and we propose a new approach to exploit efficiently the network energy, by reducing the energy consumed for cluster forming.
\end{abstract}

Keywords: Wireless Sensor Networks, Clustering Protocol, Energy Efficiency, Cluster-Head Selection, Information Routing

\section{Introduction}

Wireless Sensor Networks can offer unique benefits and versatility with respect to low-power and low-cost rapid deployment for many applications, which do not need human supervision. The nodes in WSNs are usually battery operated sensing devices with limited energy resources and replacing or replenishing the batteries is usually not an option. Thus energy efficiency is one of the most important issues and designing power-efficient protocols is critical for prolonging the lifetime. The latest developments in time critical, low cost, long battery life, and low data rate wireless applications have led to work on WSNs. These WSNs have been considered for work in certain applications with limited power, reliable data transfer, short communication range, and reasonably low cost such as industrial monitoring and control, home automation and security, and automotive sensing applications [1]. The WSNs consist of a set of sensors that communicate with each other to form a sensor field. These large numbers of nodes, which have the ability to communicate wirelessly, to perform limited computation, and to sense their surroundings, form the WSNs [2]. Specific functions can be obtained through cooperation between these nodes: functions such as sensing, tracking, and alerting [3]. These functions make these wireless sensors very useful for monitoring natural phenomena, environmental changes, controlling security, estimating traffic flows, monitoring military application, and tracking friendly forces in the battlefields.

For this work, we make some assumptions:

1) The network area is $M \times M$.

2) The number of the network nodes is $N$.

3) The base station is located in the center of the network.

4) The data packets length is $L$ bits.

5) All network nodes can reach the Base Station.

6) The clustered nodes transmit to their cluster-head, and the not clustered nodes transmit directly to the sink.

7) And, the traffic generation model is supposed a uniform event generation which mean that every sensor has a data packet to be reported in a fixed time or round.

The remainder of this paper is organized as follows. In Section 2, we briefly review related work. Section 3 describes the energy consumption model. Section 4 presents the detail of our algorithm. Section 5 shows the performance of the proposed algorithm by simulations and compares it with literature technique. Finally, Section 6 gives concluding remarks and provides some future work. 


\section{Related Work}

In order to enhance the network lifetime by the period of a particular mission, many routing protocols have been devised. One of these is network clustering, in which network is partitioned into small clusters and each cluster is monitored and controlled by a node, called Cluster Head $(\mathrm{CH})$. These cluster heads can communicate directly with the base station (BS). Other nodes send the data, sensed from the environment to these CHs. CHs first aggregate the data from the multiple sensor nodes, and then finally send it directly to the BS. Hence the $\mathrm{CH}$ should be powerful, closer to the cluster-centroid a less vulnerable [4]. Heinzelman et al. proposed LEACH [5] a protocol based on network clustering. Each cluster has a cluster-head that aggregates all the data received from the near nodes and send them to the base station. The cluster-head are selected following a distributed algorithm for each round. The [6] proposed an algorithm called TB-LEACH which is an improvement of the LEACH one. This algorithm permits to dominate the number of clusters heads to have at any transmission round, the optimal cluster-heads amount that modifies the cluster-head selection algorithm to improve the partition of cluster. This algorithm assumes that all nodes receive the messages broadcasted by the nodes selected as cluster-heads. On one hand, if a node is not reachable by a cluster head it assumes that the number of clusters heads is insufficient, and elects them to be cluster head, therefore the number of cluster-heads may be not dominated, on the other hand, this is not real with the large networks because the those messages can not reach all the network. PEGASIS [7] is an improvement of the LEACH protocol. Rather than forming multiple clusters, PEGASIS forms chains from sensor nodes so that each node transmits and receives from a neighbor and only one node is selected from that chain to transmit to the base station (sink). Gathered data moves from node to node, aggregated and eventually sent to the base station. The chain construction is performed in a greedy way. However, PEGASIS introduces excessive delay for distant node on the chain. In addition the single leader can become a bottleneck. All the previous techniques are used for homogenous networks where all the nodes have the same initial battery energy. SEP [8] is a proposed scheme for heterogeneous wireless sensor networks, which is composed of two types of nodes according to the initial energy. The advance nodes are equipped with more energy than the normal nodes at the beginning. This technique prolongs the stability period, which is defined as the time until the first node failure.

DEEC [9] is a distributed clustering scheme for heterogeneous wireless sensor networks. In DEEC the cluster-heads are elected by a probability based on the ratio between residual energy of each node and the average energy of the network. The epochs of being cluster-heads for nodes are different according to their initial and residual energy. The nodes with high initial and residual energy will have more chances to be the cluster-heads than the nodes with low energy. In the last cited works, for each round, a new cluster-heads are chosen, so, many control messages are exchanged between these $\mathrm{CHs}$ and their closest nodes to form the clusters. These control messages makes some energy lost.

\section{Energy Consumption Model}

Recently, there is a significant amount of work in the area of building low-energy radios. For the purpose of this study we use similar energy model and analysis as proposed in [5] as shown in Figure 1. For the experiments described here, both the free space $\left(d^{2}\right.$ power loss) and the multi path fading ( $\mathrm{d}^{4}$ power loss) channel models were used, depending on the distance between the transmitter and the receiver. If the distance is less than a threshold, the free space (fs) model is used; otherwise, the multi path (mp) model is used.

Thus, to transmit an $L$ - bits message over a distance d, the radio expends (1):

$$
E_{T X}(l, d)=E_{T X-\text { elec }}(l)+E_{T X-a m p}(l, d)
$$

And then:

$$
E_{T X}(l, d)=\left\{\begin{array}{lc}
l E_{\text {elec }}+l \varepsilon_{f s} d^{2} & \text { if } d<d_{0} \\
l E_{\text {elec }}+l \varepsilon_{m p} d^{4} & \text { if } d \geq d_{0}
\end{array}\right.
$$

where the threshold $\mathrm{d}_{0}$ is done by (3):

$$
d_{0}=\sqrt{\frac{\varepsilon_{f s}}{\varepsilon_{m p}}}
$$

The electronics energy $E_{\text {elec }}$ depends on many factors such as the digital coding, the modulation, the filtering, and the spreading of the signal, whereas the amplifier energy, $\varepsilon_{f s} d^{2}$ or $\varepsilon_{m p} d^{4}$, depends on the distance to the receiver and the acceptable bit-error rate.

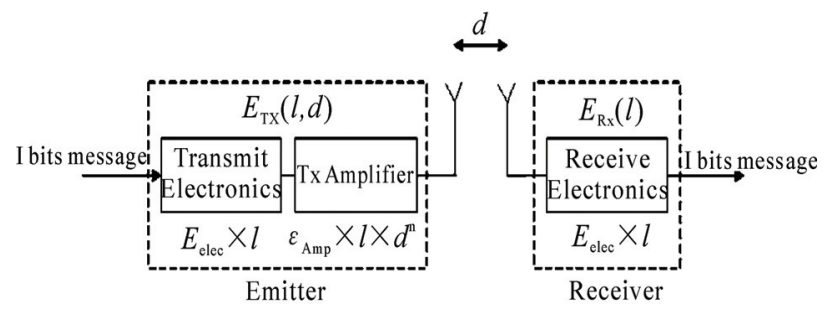

Figure 1. Radio energy dissipation model. 
To receive an 1-bit message, the radio expends (4):

$$
E_{R X}(l)=E_{R X-\text { elec }}(l)=l E_{\text {elec }} .
$$

It is also assumed that the radio channel is symmetric, which means the cost of transmitting a message from $A$ to $B$ is the same as the cost of transmitting a message from $B$ to $A$. The used parameter values in our work are given in the following Table 1.

\section{Clustering Technique for Routing in Wireless Sensor Networks}

All proposed clustering techniques in literature, use a cluster head rotation in order to balance the transmission energy cost over the network nodes, because the cluster head role is energy expansive. That permits to grant approximately, the same lifetime until the battery energy depletion. So, in every transmission round, some new nodes play concurrence to be elected as cluster head. Each node selected, has to advertise its status to its neighbor nodes, to know the nodes which will belong to its cluster and to schedule the TDMA intervals [5]. Then, some energy is consumed in this state. This energy for clustering control is considerable, and it is important to reduce this energy to use it to exploit the total network energy to extend the network lifetime.

Our contribution consists in reducing the control energy for cluster formation by keeping each selected cluster head for more than one transmission round. So, each node selected as cluster head, play this role for $\mathrm{m}$ consecutive transmission rounds before conceding it for upcoming selection nodes. The proposed algorithm, called Clustering Technique for Wireless Sensor Networks (CTRWSN) is a self-organizing, dynamic clustering method that divides dynamically, the network on a number of a priori fixed clusters. Each cluster has one cluster-head. In this work, we use two-level heterogeneous networks, in which there are two types of sensor nodes: the advanced nodes and normal nodes. Let $E_{0}$ the initial energy of the normal nodes and, $f$ the fraction of the advanced nodes, which own a times

Table 1. Radio parameter values.

\begin{tabular}{lcc}
\hline \multicolumn{1}{c}{ Description } & Symbol & Value \\
\hline $\begin{array}{l}\text { Energy consumed by the amplifier } \\
\text { to transmit at a shorter distance }\end{array}$ & $\varepsilon_{f s}$ & $10 \mathrm{pJ} / \mathrm{bit} / \mathrm{m}^{2}$ \\
$\begin{array}{l}\text { Energy consumed by the amplifier } \\
\text { to transmit at a longer distance }\end{array}$ & $\varepsilon_{m p}$ & $0.0013 \mathrm{pJ} / \mathrm{bit} / \mathrm{m}^{4}$ \\
$\begin{array}{l}\text { Energy consumed in the electron- } \\
\text { ics circuit to transmit or receive the } \\
\text { signal }\end{array}$ & $E_{\text {elec }}$ & $50 \mathrm{~nJ} / \mathrm{bit}$ \\
$\begin{array}{l}\text { Energy consumed for beam form- } \\
\text { ing }\end{array}$ & $E_{D A}$ & $5 \mathrm{~nJ} / \mathrm{bit} / \mathrm{signal}$ \\
\hline
\end{tabular}

more energy than the normal ones. Thus there are $f . N$ advanced nodes equipped with initial energy of $(1+a) E_{0}$ and $(1-f) N$ normal nodes equipped with initial energy of $E_{0}$.

We can compute the total initial energy of the networks which is given by:

$$
E_{\text {total }}=N(1-f) E_{0}+N f(1+a) E_{0} .
$$

The node $n$ becomes cluster-head for $t_{n}$ rounds. In homogenous networks, to guarantee that there are average $P_{\text {opt }} N$ cluster-heads every round, LEACH let each node $n$ becomes a cluster-head once every $t_{n}=1 / P_{\text {opt }}$ rounds. The network nodes will have different residual energy when the network evolves.

If the rotating epoch $t_{n}$ is the same for all the nodes as proposed in LEACH, the energy will be not well distributed and the low-energy nodes will die more quickly than the high-energy nodes. DEEC protocol; choose different $t_{n}$ based on the residual energy $E_{n}(r)$ of node $n$ at round $r$.

The probability threshold that each node $n$ use to determine whether itself to become a cluster-head in each round, is given as follow Equation (5):

$$
T(n)=\left\{\begin{array}{cl}
\frac{p_{n}}{\left(1-p_{n} \cdot\left(r \bmod \left(\frac{1}{p_{n}}\right)\right)\right)} & \text { if } n \in G \\
0 & \text { Otherwise }
\end{array}\right.
$$

where $G$ is the set of nodes that are eligible to be cluster-heads at round $r$.

If node $n$ has not been a cluster-head during the most recent $\frac{1}{p_{n}}$ rounds, we have $n \in G$. The $p_{n}$ parameter is given by Equation (6) from the [9].

$$
p_{n}= \begin{cases}\frac{p_{o p t} E_{n}(r)}{(1+a f) \bar{E}(r)} & \text { if } n \text { is a normal node } \\ \frac{(1+a) p_{\text {opt }} E_{n}(r)}{(1+a f) \bar{E}(r)} & \text { if } n \text { is an advanced node }\end{cases}
$$

where $E_{n}(r)$ is the residual energy of the node $n$ at the round $r, \bar{E}(r)$ denotes the average energy of the network at round $r$, which can obtained by (7)

$$
\begin{gathered}
\bar{E}(r)=\frac{E_{\text {total }}}{N}\left(1-\frac{r}{R}\right), \\
R=\frac{E_{\text {total }}}{E_{\text {round }}},
\end{gathered}
$$




$$
E_{\text {round }}=L\left(2 N E_{\text {elec }}+N E_{D A}+k \varepsilon_{m p} d_{\text {toBS }}^{4}+N \varepsilon_{m p} d_{\text {toCH }}^{2}\right) \text {, }
$$

$k$ is the number of clusters, $E_{D A}$ is the data aggregation cost which is expended in the cluster-heads, $d_{t o B S}$ is the average distance between the cluster-head and the base station, and $d_{t o C H}$ is the average distance between the cluster members and the cluster-head. If the nodes are uniformly distributed, from $[5,10]$ we can give:

$$
d_{t o C H}=\frac{M}{\sqrt{2 k \pi}} \text { and } d_{t o B S}=0.765 \frac{M}{2} .
$$

By the Equation (9), we can find the optimal value of $k$ that minimizes $E_{\text {round }}$, which is (10):

$$
k_{\text {opt }}=\frac{\sqrt{N}}{\sqrt{2 \pi}} \frac{\sqrt{\varepsilon_{f s}}}{\sqrt{\varepsilon_{m p}}} \frac{M}{d_{t o B S}^{2}} .
$$

This value of $k$ is used to determine $E_{\text {round }}$, and therefore, by (7) and (8) each node $n$ can find the value of the parameter $p_{n}$ used in $T(n)$ calculation.

And, for each round $r$, when node $n$ finds it is eligible to be a cluster-head, it will choose a random number between 0 and 1 . If the number is less than threshold $T(n)$, the node $n$ becomes a cluster-head during the current round. The operation of CTRWSN is broken up into rounds where each round consists of a set-up phase and steady-state phase. In the following sub-sections we will detail each of these phases.

\subsection{A Set-Up Phase}

Every transmission round, each node $\mathrm{n}$ uses the Formula (5) to calculate the $T(n)$ value and choose a random number between 0 and 1 . If this chosen number is less than the calculated threshold $T(n)$, the node $n$ becomes a cluster-head. The selected cluster heads broadcast an advertisement message to the network to declare themselves as cluster heads. Receiving this message, the not cluster head nodes belong to the cluster which the energy to join is minimum among all selected cluster-heads. The node can determine the needed energy to transmit to the cluster head based on the received signal strong.

Once the nodes decide to which cluster belong, they inform the cluster-head transmitting a join-request message to it, using CSMA/CA MAC protocol.

A header, the node ID and the cluster-head ID, forms this message, which is a short one. This message size grants to reduce the time channel access and the transmission energy cost. Receiving all nodes joinmessages, the cluster-head schedule a TDMA allocating a slot time to each cluster's nodes. After that, the cluster nodes are informed by a broadcasted message containing the TDMA schedule. The choice of TDMA technique in the cluster allows a energy saving, because no collisions caused and the node can pass to sleep mode when it is not transmitting; in this way, the clusters are formed.

\subsection{Steady-State Phase}

Once the clusters are established, the nodes transmit their data messages towards the cluster-head. Within the cluster, the communication uses TDMA, as described in the set up phase. When the cluster-head receives all the nodes data, it performs its compression, to form a new message that sent to the base station.

Figure 2 gives the flowchart that explains the work of the proposed algorithm.

The network function is divided into cycles, each cycle lasts for $\mathrm{m}$ transmission rounds. Then, selected nodes for cluster-heads play this role for $m$ consecutive transmission rounds.

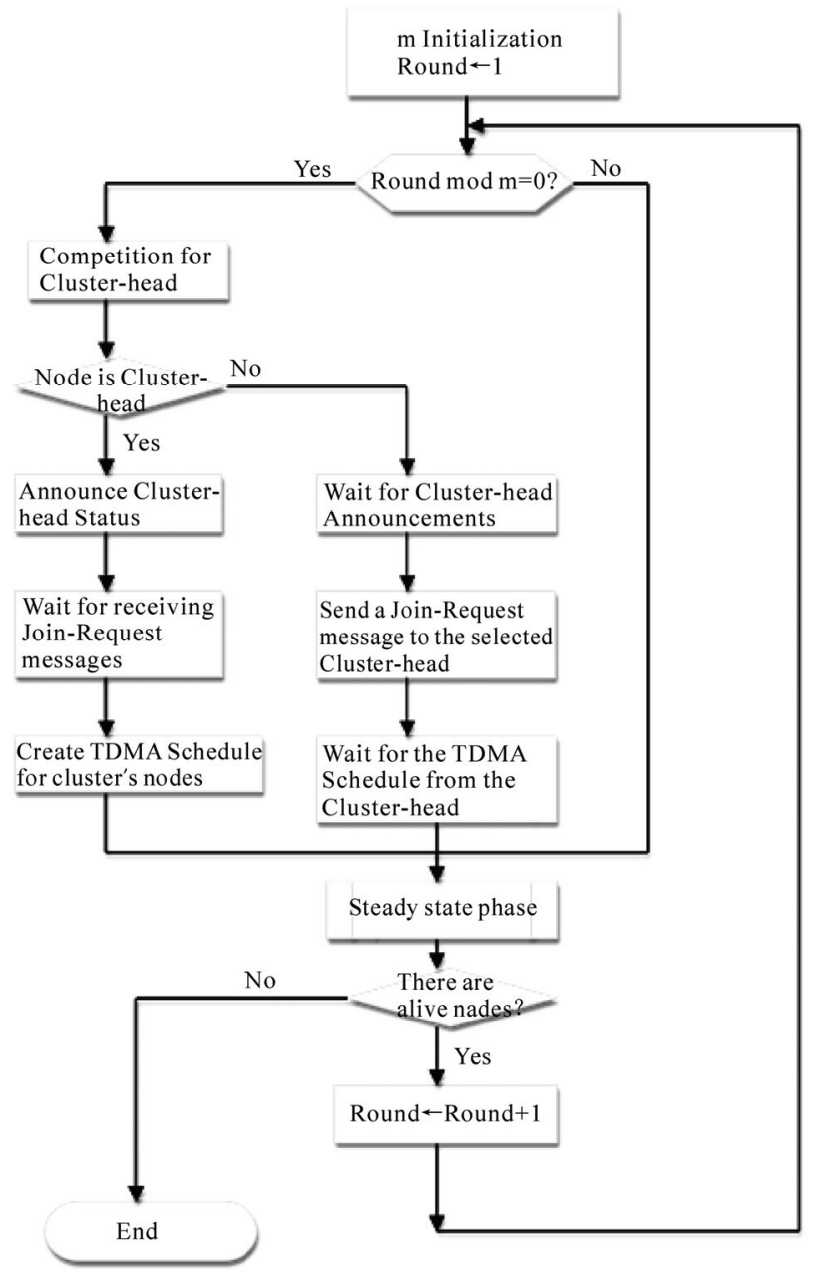

Figure 2. Protocol flowchart. 
It is so difficult to determine analytically the parameter $m$, because the nodes deployment is random and the cluster-heads position is also stochastic, then we determine this optimal value based on simulation.

\section{Simulation and Results}

The simulation parameters values used in our work are given in the Table 2 .

For the simulation bellow, we fix $\mathrm{f}=0.2$ and $\mathrm{a}=3$. Figure 3 represents the DEEC energy consumed in the network for the clusters forming for every transmission round. This energy is expended by the messages exchanged between the cluster-heads and its belonging nodes to form the clusters. As we can see, a lot of the network energy is lost to control the clusters formations. The total of this energy is evaluated as $33.3824 \mathrm{~J}$ which is $41.73 \%$ of the total network energy, and in average $0.0057 \mathrm{~J}$ per round.

To find the optimal value of the parameter $m$, we do simulation by varying it. Figure $\mathbf{4}$ gives the relative network lifetime. As depicted in this figure, the relative network lifetime becomes approximately constant when the parameter $m$ is greater than 10. This lifetime is maximal when the cluster-head duration $\mathrm{m}$ is equal to 32 ; each node selected as cluster-head, plays this role for $\mathrm{m}$ consecutive transmission rounds that permits to economize the energy consumed by the cluster-heads to form their clusters, because the number of control messages is reduced. As we can see in the figure, if the cluster-head period duration becomes longer than a threshold, even if the network control energy is reduced, oppositely, the network lifetime decreases slightly. The reason of that is the unbalancing of the cluster-heads energy load over the network nodes. So, some nodes are highly solicited when they belong to a cluster. Continuing to belong to the same cluster, some unlucky nodes have to transmit for larger distance, and then there energy drains quickly. We can see in Figure 5 the energy for forming the clusters in the CTRWSN. Figure 6 gives the network lifetime of the proposed protocol compared to DEEC one. The first node dies in the 718 th round but in CTRWSN it occurs in the 1271st

Table 2. Simulation parameter values.

\begin{tabular}{lcc}
\hline \multicolumn{1}{c}{ Description } & Symbol & Value \\
\hline Network dimension & $M$ & $100 \mathrm{~m}$ \\
Number of network nodes & $N$ & 100 \\
Data packet length & $L$ & $4000 \mathrm{bits}$ \\
Control packet length & $L_{c r t}$ & $200 \mathrm{bits}$ \\
$\begin{array}{l}\text { Optimal probability } \\
\text { Advanced Nodes percentage }\end{array}$ & $p_{\text {opt }}$ & 0.1 \\
$\begin{array}{l}\text { Fraction of advanced nodes energy } \\
\text { to normal nodes }\end{array}$ & $f$ & Variable \\
\hline
\end{tabular}

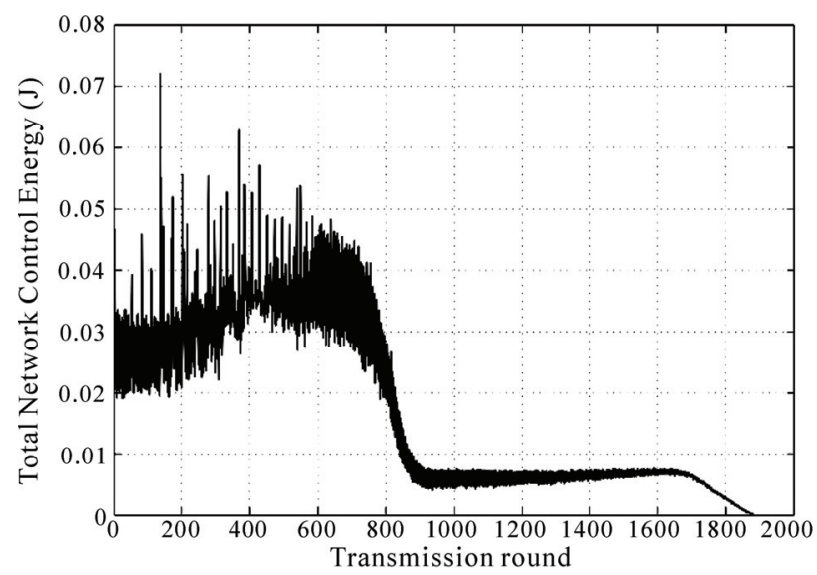

Figure 3. Total network energy for Clusters forming in DEEC.

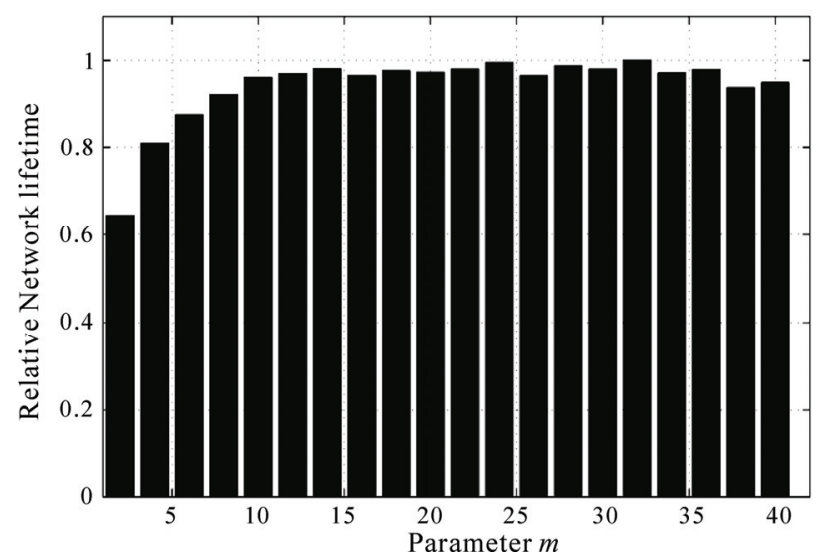

Figure 4. Relative network lifetime.

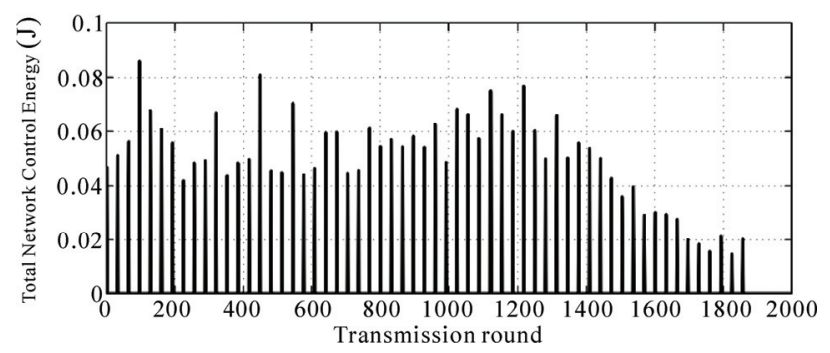

Figure 5. Total network energy for clusters forming in CTRWSN.

round, which means that the stable region is extended by up to $77 \%$. The half network nodes die in DEEC at the 832nd round and in CTRWSN it happens in the 1898th round and the last node die in the 5865th in DEEC, and 9494th round in CTRWSN, which is approximately $62 \%$ longer than DEEC.

Figure 7 gives the network remaining energy. The average network remaining energy in DEEC is $10.2149 \mathrm{~J} /$ round and in CTRWSN is 13.9502 /round that means that the CTRWSN consumes little energy compared to DEEC, which helps to extend the network lifetime for many extra-rounds. 
Figures 8(a) and 8(b) gives the network lifetime defined until the first node dies when $a$ and $f$ are varying.

This figure shows that the proposed technique provides best results when the network parameters change.

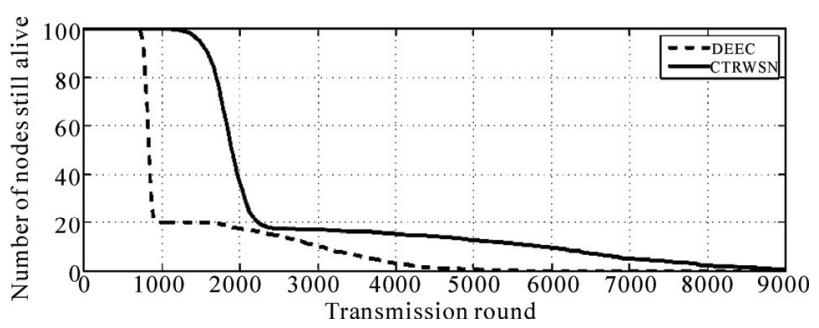

Figure 6. Network lifetime comparison.

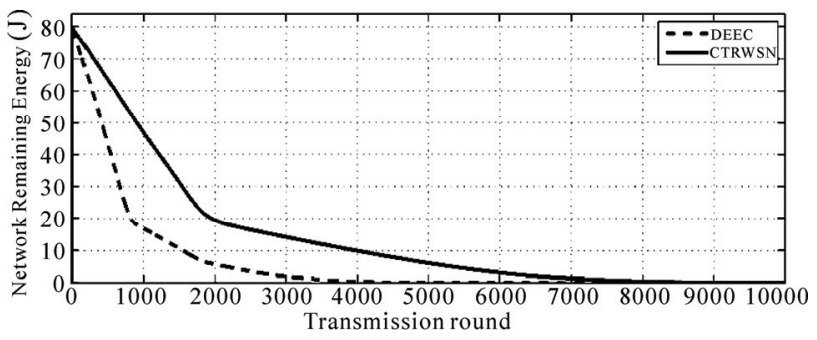

Figure 7. Network remaining energy comparison.

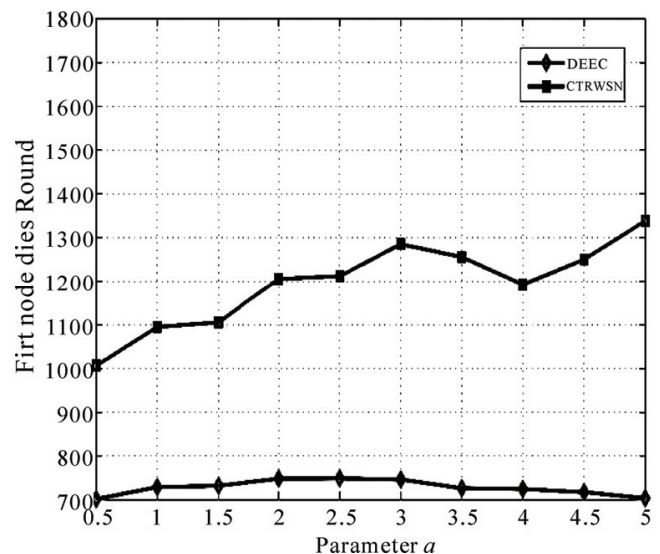

(a)

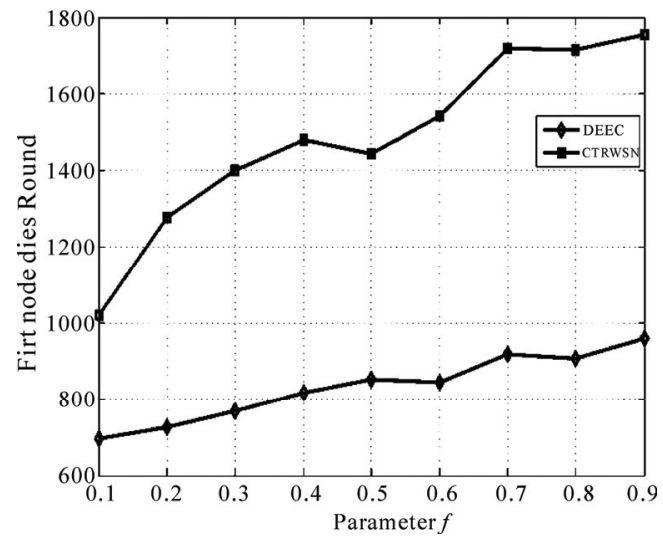

(b)

Figure 8. Network lifetime until the first node dies. (a) $f=0.2$ and $a$ varying from 0.5 to 5 ; (b) $a=3$ and $f$ varying from 0.1 to 0.9 .

\section{Conclusions and Future Works}

In this paper, we have proposed a clustering based routing protocol for heterogeneous WSNs, which is entirely distributed. We have interested in reducing the number of control message, and so, the protocol overhead.

Through the simulation, we demonstrate that the proposed algorithm allows a large stable network lifetime compared to the most known clustering algorithms in this area. As future work, we will reconsider the probability of becoming cluster-head, to extend yet the network lifetime.

\section{Acknowledgements}

This work was supported by the Hassan II Académie des Sciences et Techniques.

\section{References}

[1] F. Akyidiz, W. Su, Y. Sankarasubramaniam, and E. Cayirci, "Wireless sensor network: A survey," Computer Networks, Vol. 38, No. 4, pp. 393-422, 2002.

[2] K. Romer, O. Kastin, and F. Mattern, "Middleware challenges for wireless sensor networks," ACM SIGMOBILE Mobile Computing and Communications Review, Vol. 6, No. 4, pp. 59-61, 2002.

[3] R. Shorey, A. Ananda, and W. T. Ooi, "Mobile, wireless, and sensor networks," 1st edition, IEEE Press, John Wiley \& Sons, 2006.

[4] Z. Khalid, G. Ahmed, N. M. Khan, and P. Vigneras, "A real-time energy-aware routing strategy for wireless sensor networks," Asia-Pacific Conference on Communications, Bangkok, Thailand, pp. 381-384, 2007.

[5] W. R. Heinzelman, A. P. Chandrakasan, and H. Balakrishnan, "An application-specific protocol architecture for wireless microsensor networks," IEEE Transactions on Wireless Communications, Vol. 1, No. 4, pp. 660-670, 2002.

[6] J. Hu, Y. Jin, and L. Dou, "A time-based cluster-head selection algorithm for LEACH", Proceeding of IEEE Symposium on Computers and Communications, Marrakech, Morocco, 6-9 July 2008.

[7] S. Lindsey and C. S. Raghavendra, "PEGASIS: Power efficient gathering in sensor information systems," Proceedings of the IEEE Aerospace Conference, Big Sky, Montana, March 2002.

[8] G. Smaragdakis, I. Matta, and A. Bestavros, "SEP: A stable election protocol for clustered heterogeneous wireless sensor networks," Second International Workshop on Sensor and Actor Network Protocols and Applications, 2004.

[9] L. Qing, Q. X. Zhu, and M. W. Wang, "Design of a distributed energy - efficient clustering algorithm for heterogeneous wireless sensor networks," Computer Communication, Elsevier, Vol. 29, No. 12, pp. 2230-2237, 2006.

[10] S. Bandyopadhyay and E. J. Coyle, "An energy efficient hierarchical clustering algorithm for wireless sensor networks," Proceeding of International Conference on Computer Communication, Vol. 3, pp. 1713-1723, April 2003. 